\title{
Optics related courses in research and education center Plasma at Petrozavodsk State University
}

A. Khakhaev, L. Luizova, A. Moschevikin

A. D. Khakhaev, L. A. Luizova, A. P. Moschevikin, "Optics related courses in research and education center Plasma at Petrozavodsk State University," Proc. SPIE 9663, Eighth International Topical Meeting on Education and Training in Optics and Photonics, 966315 (6 October 2003); doi: $10.1117 / 12.2207485$

SPIE Event: Eighth International Topical Meeting on Education and Training in Optics and Photonics, 2003, Tucson, Arizona, United States 


\title{
Optics related courses in Research and Educational Center "Plasma" at Petrozavodsk State University
}

\author{
A.D. Khakhaev, L.A. Luizova and A.P. Moschevikin \\ Petrozavodsk State University, 33 Lenin Str., Petrozavodsk, 185640, Russia \\ rec@plasma.karelia.ru
}

\begin{abstract}
The educational and research facilities in optics provided by the Research and educational center "Plasma" are described. Students study traditional, laser and correlation spectroscopy; interference and holography; image processing; nonlinear optics as well as methods of automated optical data acquisition and processing. The main attention in lectures, laboratory training and student's individual research works is attracted to optical methods applied to plasma diagnostics.

(C)2003 Optical Society of America

OCIS codes: (000.2060) Education, (350.5400) Plasmas
\end{abstract}

\section{Introduction}

The research and educational center on basic problems of application of low temperature plasma physics (REC-013 "Plasma", Petrozavodsk, Russia) was found in 2002 within the project supported by the US Civilian Research and Development Foundation, Ministry of Education of Russian Federation and government of Republic of Karelia.

This project consists mainly of the development of experimental and theoretical investigations in the field of atomic and molecular physics, low temperature plasma physics and relevant basic applications. The main attention is attracted to interconnection between fundamental properties of "new" objects of atomic and molecular physics and low temperature plasma physics on the one hand and behavior of these objects in operating conditions of practically important devices on the other hand. Term "new" objects stands for complex ions, Rydberg atoms and molecules, clusters, fullerenes, nanotubes, plasma crystals. Experimental investigations of physical and chemical properties of these objects as well as mechanisms of their formation and destroy in low temperature plasma conditions are connected with the development and modification of beam, optical, radio-frequency and X-ray methods of control and diagnostics of plasma media with use of modern laser techniques, spectral analytical setups and modem vacuum and cryogenic equipment and involve the computer processing of measured data. The results of the investigations are supposed to be used in exploration and development of new knowledge in theoretical physics, micro- and nanoelectronics, and also for design of new types of electronic devices and plasma technologies.

The majority of modern ways of control of conditions and characteristics of plasma objects and results of interaction of plasma with substance is based on analysis of spectral and spatial distribution of radiation, emitted, absorbed or scattered by object of investigation. Undertaking of such studies requires high professionalism and high level of experimental technology and installations. Training specialists on optical and x-ray diagnostics and control of characteristics, conditions and structures of materials and substances is traditionally carried out at physical technical faculty. REC "Plasma", from the one hand, formulates problem-oriented tasks for these specialists and, from another, provides new instrumentation and new abilities in methods development. In addition to geometric and physical optics which are included in course of basic physics, the theoretical basis of training in the sphere of optics is establishing in course of quantum physics, where significant attention is attracted to the questions of theory of atomic spectra, forming spectral lines shapes, especially in plasma conditions, as well as to the questions of quantum optics, including photon statistics, interference of quantum states and interference of intensities. One of the courses in training program is laser physics. Also applications of optical methods to plasma diagnostics resulted in special course supported by laboratory classes. For practical application of basic knowledge formed by these theoretical courses there have been introduced a special set of laboratory educational-research programs connected with certain studied problems.

\section{Optical methods of plasma diagnostics}

The purpose of this course and accompanying it laboratory practical programs - not only to arm the student by instruments and facilities of plasma diagnostics, but also to maintain and to teach to apply in practice fundamental knowledge, obtained at study of wave and geometric optics, quantum and laser physics, math basis.

Preamble part of course describes the well-known models of plasma (for instance, model of full or local thermodynamic balance, kinetic model) and shows the parameters to be determined and experiments that can 
confirm or disprove validity of these models. After that a general scheme of diagnostics is stated, in which it is shown that any method of diagnostics can be introduced, only if the following parameters and factors are known.

1. A physical model linking required local and instant values of parameters of plasma in a certain elementary volume (densities of electrons $\mathrm{N}_{\mathrm{e}}$, ions $\mathrm{N}_{\mathrm{i}}$, atoms and molecules of different sorts both in ground $\mathrm{N}_{\mathrm{a}}$ and excited states $\mathrm{N}_{\mathrm{a}}{ }^{*}$, electronic $\mathrm{T}_{\mathrm{e}}$ and atomic $\mathrm{T}_{\mathrm{a}}$ temperatures and electric field intensity $\mathrm{E}$ ) with optical parameters of elementary volume: radiation $\varepsilon(\lambda)$ and absorption $\mathrm{k}(\lambda)$ factors in spectral lines and continuous spectrum, refractive index $n(\lambda)$, as well as differential cross section of scattering $\sigma(\lambda)$ depending on wavelength and integral characteristics of radiation I and absorption $\mathrm{K}$ in spectral lines. In case of plasma with macroparticles, their contribution to the characteristics mentioned above should be taken into consideration apart.

2. A source model allowing to interconnect the optical features of source (plasma object): spectral energy radiance of source surface $b(\lambda)$, optical thickness $\tau(\lambda)$, portion of falling radiation $P(\theta, \lambda)$, scattered in a certain angle $\theta$ to the direction of radiation propagation, phase difference of incoming wave $\Phi(\lambda)$ or deflection of beam from initial direction $\alpha$ at passing through plasma with characteristics of elementary volumes. (The simplest interconnection of these parameters is in case of homogeneous source, more complicated - for axial symmetric heterogeneous source and even more complicated - for free type of heterogeneity. These problems are discussed in corresponding to sections of course.)

3. A project for laboratory equipment, providing conversion of optical characteristics to data array of registering system.

On the basis of chosen physical models and data acquired in experiment a researcher carries out the conversion of data array to spatial-temporal distribution of required plasma parameters. It is called as "inverse problem", which solution can be unstable due to experimental inaccuracy. The way of overcoming these difficulties is considered in special section of course.

Section "Spectroscopic methods of diagnostics of plasma" is devoted firstly to the classical theory of interconnection of integral intensity of spectral lines and integral absorption with quantum states population and secondly to relation of intensity of continuous spectrum background with electron density and temperature [1,2]. The theory of spectral line broadening as well as conditions under which different broadening factors can be considered as independent are studied [3]. Then the resulting line shape is a convolution of profiles, defined by various reasons. If all broadening factors except one may be neglected then basing on the form of profile it can be determined either atomic temperature (Doppler broadening) or electron density (Stark broadening) or ground state atoms density (resonant or Van-der-Waals broadening). In case of joint influence of several broadening factors their contribution can be separated if they lead to different dependencies of radiation factors on wavelength. For instance, if Doppler line shape is

$$
\varphi_{D}(\lambda)=A \cdot \exp \left[-\beta\left(\lambda-\lambda_{0}\right)^{2}\right], \beta=c^{2} M /\left(2 \chi \lambda_{0} T_{a}\right),
$$

where $\mathrm{c}$ - light velocity, $\mathrm{M}$ - mole mass, $\chi$ is the Boltzmann constant, $\lambda_{0}$ - spectral line center, and resonance line shape is [3]

$$
\varphi_{\mathrm{R}}(\lambda)=\mathrm{B} /\left[\left(\lambda-\lambda_{0}\right)^{2}+\gamma^{2}\right], \quad \gamma=\lambda_{0}^{2} \lambda_{\mathrm{R}} \mathrm{e}^{2} \mathrm{fN}_{\mathrm{a}}\left(\mathrm{g}_{0} / \mathrm{g}_{R}\right)^{1 / 2} /\left(4 \pi \mathrm{mc}^{2}\right),
$$

where $\mathrm{m}, \mathrm{e}$ - electron's mass and charge, $\lambda_{\mathrm{R}}, \mathrm{f}$ - wavelength and strength of oscillator of transition, connecting one of states of this line with ground state, $\mathrm{g}_{R}-$ stat. weight of this level, $\mathrm{g}_{0}-$ stat. weight of ground state, then according to convolution theorem:

$$
\mathrm{F}(\omega)=\Phi\{\varphi(\lambda)\}=\sqrt{2 \pi} \Phi\left\{\varphi_{\mathrm{D}}(\lambda)\right\} \cdot \Phi\left\{\varphi_{R}(\lambda)\right\},
$$

where $\varphi(\lambda)$ is the resulting line shape and $\Phi$ is Fourier transform operator. In our case

$$
\ln (F)=-\omega^{2} /(4 \beta)-\gamma|\omega|+C
$$

A, B, C in equations 1,2,4 - unimportant constants. From the system of equations (4) values of $\beta$ и $\gamma$ may be found by root mean square method for several values of $\omega$.

Type of line shape, integral intensity and absorption factor can be easily calculated from values of radiance of source surface and optical thickness only in case of homogeneous source. In other cases these characteristics must be determined for different points of surface or for different directions of exposure; spatial distribution of the optical characteristics of elementary volumes is derived by means of solving corresponding integral equations. Anyway spectral distribution of radiance of surfaces and optical thickness in one or several spatial points must be measured. Therefore main part in this course is devoted to special spectral instruments. For each type of these instruments (prism, diffraction and Fourier spectrometers, Fabry-Perot interferometers) there have been studied the factors, depending on working range and best resolution, i.e. on form and width of apparatus spread function. It is shown 
principle advantage of interferential instruments to slit apparatus in study of weak light sources. Few methods of realization of image spectrometer what is necessary in investigations of spatially heterogeneous sources are considered. Aside from trivial variants, containing different systems of spatial scanning, as well as optical fiber system, separating radiation from different parts of the source on different spectral nodes, modern, often of original design, devices based on multiple-unit photodetectors (CCD arrays and matrices) are described.

To investigate one-dimensional distribution of radiance it is possible to use a slit spectrometer with photosensitive matrix. Direction of change in radiance should correspond to the axis of slit and to columns of matrix, each line of matrix will register the spectrum of a certain spatial element.

At study of objects with "rare" line spectrum good result can be given by well known in astronomy slitless spectrometer. Placing an investigated object or its image at the entry of usual prism or diffractional spectrometer instead of input slot (fig. 1), the picture of object in light of different wavelengths will be registered at the output of this instrument. The condition of "no superposition" of different spectral images is:

$$
\Delta \lambda_{\min }>\mathrm{t}(\mathrm{d} \lambda / \mathrm{dx}) \text {, }
$$

where $\Delta \lambda_{\min }$ - minimal spectral distance between lines, $\mathrm{t}$ - size of object in the direction of dispersion of instrument, $\mathrm{d} \lambda / \mathrm{dx}$ - its inverse linear dispersion.

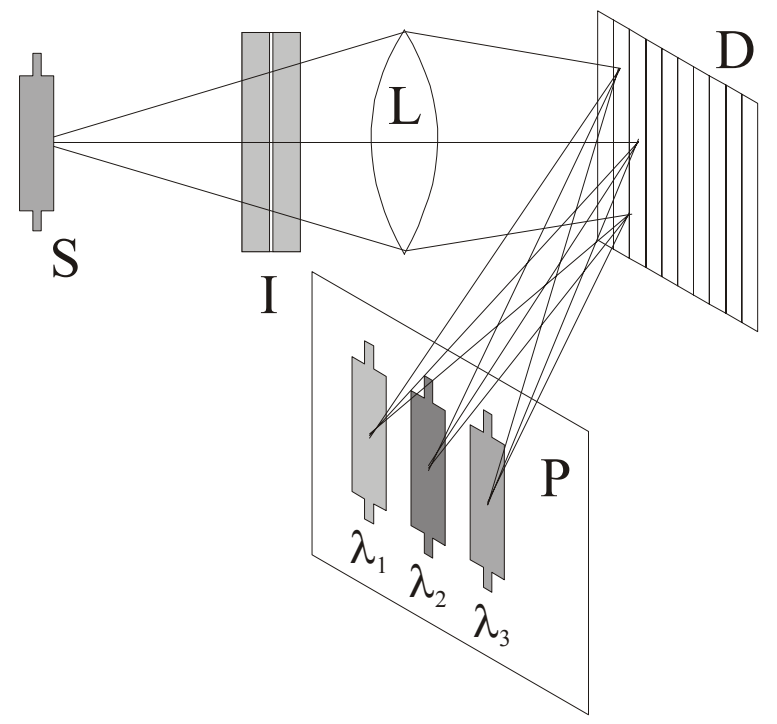

Fig. 1. Slitless spectrometer. S - source, L - lens, D - diffraction grating, P - output plane with picture of object in various wavelengths, I - fine interferometer, which is used in investigations with continuous spectrum.

This method may also be applied to study spatial distribution of radiance in different ranges of continuous spectrum. To implement this it is necessary to "thin out" the whole spectrum beforehand, i.e. to transform it in line structure with an interval between "lines" of $\Delta \lambda$. It may be done by passing radiation through fine interferometer Fabry-Perot. Students are to calculate the system for registration of spatial distribution of radiance of an object of given size with given spectral step $\Delta \lambda$, they must choose the thickness $\mathrm{d}$ of the interferometer, and also parameters of grating and focal length of lens. However it should be taken into consideration that picture of object in image plane will be blurred a little. This image blurring is determined by the passband spectral width of a filter and by dispersion of grating, nevertheless achieved in the above mentioned way spatial resolution (approximately 30 elements per width of object) as a rule is enough for practical purposes.

For stationary in time or slowly changing objects the best variant of image spectrometer can be built on the basis of interference spectrometer (Fabry- Perot with filter of preliminary monochromatization or Fourier spectrometer), since such a system combines a source image with interference rings pattern on output (fig. 2). In this case spectrum scanning is realized by means of change of optical width (base) of interferometer, and spatial resolution is limited only by quantization of matrices and is practically well enough. Here the difference between optical paths of two waves in interferometer (axial and propagating at the angle $\delta \varphi$ to it) may be neglected if the following condition is fulfilled:

$$
\delta \varphi \leq\left(2 \delta \lambda_{\mathrm{a}} / \lambda\right)^{1 / 2},
$$

where $\delta \lambda_{a}-$ spectral width of spread function of spectrometer. 


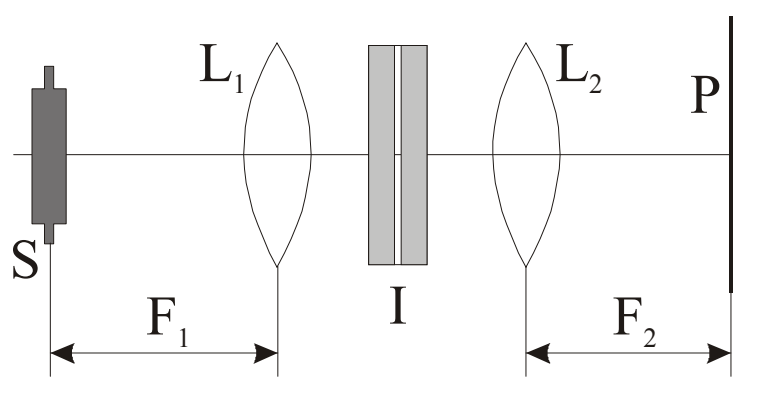

$\mathbf{a}$

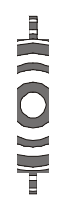

b

Fig. 2. a). Installation with Fabry-Perot interferometer (I). $S$ - source, $P$ - imaging plane, $L_{1}, L_{2}$ - lenses; b). Interference pattern in $\mathrm{P}$ plane.

For example, it is possible to obtain spatial spectrum distribution on the object of $1 \mathrm{~cm}$ size using Fourier spectrometer with $\delta \lambda_{\mathrm{a}}=1 \mathrm{~nm}$ and drawing lens with focal length of $30 \mathrm{~cm}$. For objects of large angular sizes also there are no principle difficulties. It should only be taken into account in case of processing interferograms that for points of interference pattern, distanced from the axis of system on angle $\varphi$, difference in optical paths is $\Delta=\Delta_{0} \cos \varphi$, what is less, than $\Delta_{0}$ - difference of optical paths along the axis of system.

Since photosensitive matrices are essential elements of image spectrometer, metrological features of different types of matrices: spectral sensitivity, range of linearity, noise level, deviation of sensitivity of different elements, are researched by students within the framework of laboratory educational-research programs.

The study of spectral instruments is terminated by a section, dedicated to spectroscopy of high resolution, i.e. resolution, not achievable by the most exact optical spectral instruments. Small differences in radiation frequencies and small line widths corresponding to radio frequency range may be measured by methods of optical heterodyning or methods of correlation spectroscopy (interference of intensities) [4]. The method of optical heterodyning can easily be explained in common terms of summation of coherent waves and by the following concept: photo sensor is a square-law detector. The phenomenon of interference of intensities (correlation spectroscopy) can be understood either on the basis of knowledge got in course of quantum physicists about Bose-Einstein statistics, which comply with the photons, or on the basis of classical wave theory of light in suggestion that observed radiation is a result of superposition of waves, emitted by independent sources. Each of these waves can be described by complex function, intensity is proportional to the square of module of this function and has a random nature; correlation function for two spatial-temporal points depends on degree of coherence of fields in these points. It is shown that both approaches lead to similar results. The study of this subject fastens the fundamental knowledge on physical optics and probability theory. Since radiation spectrum is a Fourier image of degree of coherence the information on spectral lines widths can be obtained from correlations intensity function. As an application to problems solving in REC "Plasma" this method is used for measurement of diffusion coefficient of dust particles in glow discharge plasma. These measurements are considered to be an imperative step of investigations of mechanisms of forming ordered structures in dust plasma [5]. On fig. 3 one of the results of work carried out with participation of student and reported at conference on Physics of Low Temperature Plasma (PLTP-03) [6] is presented.

Calculation were done using this formula:

$$
G=\frac{g(\tau)-g(\infty)}{g(0)-g(\infty)}=\exp \left(-2 q^{2} D \tau\right),
$$

where $\mathrm{g}(\tau)$ - correlation function of intensity of scattered light, D - diffusion coefficient, $\mathrm{q}$ - wave number $q=(2 \pi / \lambda) \sin (\beta / 2), \beta-$ angle of scattering, in our case $\beta=90^{\circ}$. It is obvious that experimental curve corresponds well enough to calculated curve with $\mathrm{D}=7.5 \cdot 10^{-8} \mathrm{~cm}^{2} \mathrm{~s}^{-1}$.

The spectroscopic methods of Doppler-free broadening also belong to methods of high-resolution spectroscopy. These methods allow distinguishing the broadening taking place due to interaction with charged or neutral particles in plasma and to determine their densities even if this interaction caused broadening is less than Doppler effect. In the discussed course methods of two photon spectroscopy and interference of states of atom are studied. 


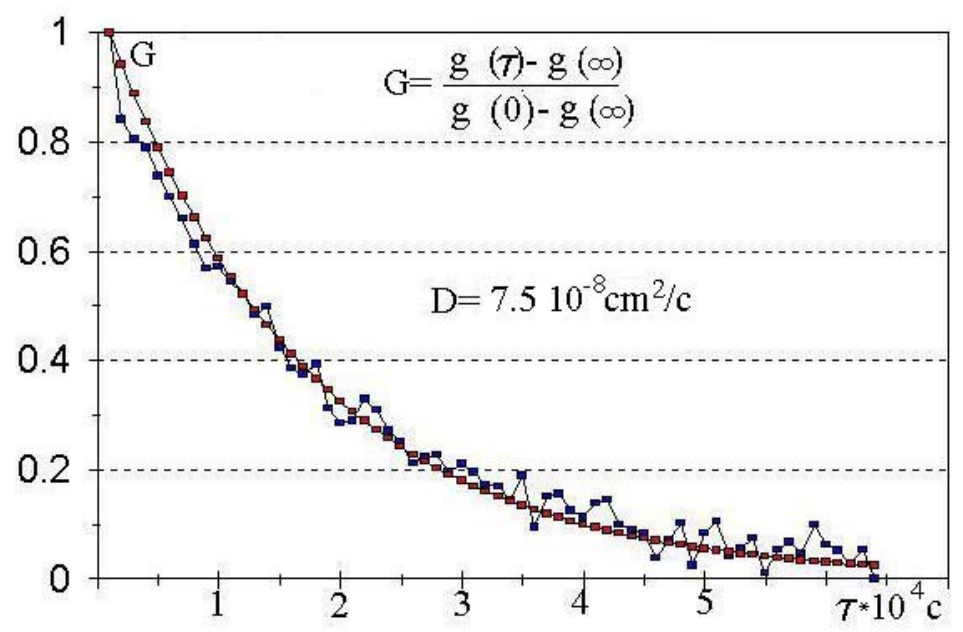

Fig. 3. Normalized correlation function of laser radiation (wavelength $\lambda=632.8 \mathrm{~nm}$ ), diffused on cloud of $\mathrm{Al}_{2} \mathrm{O}_{3}$ particles in Neon discharge at pressure of $53 \mathrm{~Pa}$ and discharge current of $2 \mathrm{~mA}$. Smooth curve corresponds to calculated normalized correlation function.

The section "Spectroscopic methods of plasma diagnostics" is terminated by the description of characteristics of photo sensors (quantum efficiency, noise level and nature of noises), analysis of factors, limiting sensitivity, spectral, temporal resolution of spectroscopic installations and also by the description of methods of measurement of absolute values of radiance of surface and optical thickness of source. There have been considered both simplest methods: illumination by tunable laser, by source of continuous spectrum and its own radiation, and well-known more sensitive methods: laser fluorescence [7] and intra resonator spectroscopy [8].

In section "diagnostics based on refractive index" the interconnection of refractive index with density of atoms and electrons in elementary volume of plasma is determined on the basis of classical theory of dispersion. Two boundary conditions are considered: 1) wavelength of probe (input) radiation is close to wavelength of an absorption line of atoms or ions presenting in plasma, 2) wavelength is far enough from all lines of atoms and ions.

In first case the density of atoms on absorbing level can be measured by methods of polarization spectroscopy. At illuminating plasma by radiation with frequency $\omega$, close to absorption frequency $\omega_{0}$ of one of the plasma component, refractive index is described by formula:

$$
n_{1}-1=\frac{q N \Delta \omega}{2 \omega\left(\Delta \omega^{2}+\gamma^{2} / 4\right)},
$$

where $\Delta \omega=\omega_{0}-\omega, N$ - density of atoms on absorbing level, $\gamma$ - spectral width of absorption line (disregarding Doppler broadening). $q=\frac{e^{2}}{2 \varepsilon_{0} m}\left(\varepsilon_{0}-\right.$ dielectric constant of vacuum).

If place is put in magnetic field, which direction matches the direction of propagation of plane-polarized wave with frequency $\omega$, then after passing through plasma polarization plane will be turned from initial direction on a certain angle $\varphi$. This occurs due to effect of line splitting. In strong magnetic field one absorption line splits it two with frequencies $\omega_{0}+\omega_{\mathrm{B}}$ and $\omega_{0}-\omega_{\mathrm{B}}$, where $\omega_{\mathrm{B}}-$ Zeeman splitting frequency, proportional to the induction of magnetic field B. Herewith plane-polarized wave with frequency $\omega$ transforms in two circularly polarized waves, propagating with different velocities. Difference in refractive indexes for these waves will be $\Delta \mathrm{n}$. It's clear that this value depends on magnetic field. It is small in case of both tiny and large splitting because at large spectral distances from $\omega_{0}$ refractive index is practically equal 1 . This phenomenon is used in different variants of the method. For example in one of the variants maximum value of angle shift is measured. This value, as it was stated in work of W.R. Bennet [9], is unambiguously connected with absorption coefficient in the center of line $\mathrm{k}\left(\omega_{0}\right)$, and, consequently, with population of a level:

$$
\beta=-0.28 k\left(\omega_{0}\right) L \sin \frac{\omega_{0}-\omega}{0.3 \Delta \omega_{D}},
$$


where $\mathrm{L}$ - length of optical path of beam in plasma, $\Delta \omega_{\mathrm{D}}-$ Doppler line width. By "waving" polarization plane of illuminating laser and determination of wave of the frequency of intensity modulation and polarized perpendicular to initial laser light, it is possible to measure the tilt of polarization plane for the angle less than $10^{-6}$ radian, what allows to register the density of absorbing atoms down to $10^{6} \mathrm{~cm}^{-3}$ in conditions of $1 \mathrm{~cm}$ optical path length and Doppler width of order of $10^{9} \mathrm{~s}^{-1}[10]$.

The second variant of polarization spectroscopy [11] assumes the work on lines with large absorption on frequencies $\omega$, distanced from the center of line $\omega_{0}$ farther than Doppler width. Plasma located in magnetic field is illuminated by polarized tunable laser radiation with $\mathrm{I}_{0}$ intensity. The intensity of light, initially polarized in perpendicular plane and passed through plasma, is registered:

$$
I=I_{0} \exp [-k(\omega) L] \sin ^{2} \varphi .
$$

The intensity as a function of frequency consists of two multiplicands, the first increases, but the second decreases with growth of $\omega_{0}-\omega$. This dependence has its maximum, when $\mathrm{x}=\omega_{0}-\omega_{\text {мax }}$, what allows to find either the density of absorbing atoms $\mathrm{N}$ if $\gamma$ is known, or linewidth $\gamma$ if $\mathrm{N}$ is known.

At far spectral distances from absorption lines the refractive index of plasma is expressed as:

$$
n-1=q \sum_{a j k} \frac{N_{a j} f_{a j k}}{\omega_{a j k}^{2}-\omega^{2}}-\frac{q N_{e}}{\omega^{2}} .
$$

The first summand contains the sum on particles of different sorts a, existing in initial state $\mathbf{j}$, from which a transition is possible to any $\mathbf{k}$ state. However in practice only ground states of atoms and transitions with frequency $\omega_{\mathrm{ajk}}$ and oscillator force $\mathrm{f}_{\mathrm{ajk}}$ are taken into consideration since these states have the largest densities. It should be mentioned that $\mathrm{f}_{\mathrm{ajk}}$ are calculated by quantum mechanics methods and are tabulated for many transitions (for example in [12]). To distinguish the contributions of electrons and atoms in the refractive index few measurements on different wavelengths should be done.

The main method of measurement is interferometry. The difference in $\theta$ (wave phase of the beam with wavelength $\lambda$ ) between two beams (passed a distance L through plasma and passed the same distance in medium with well known refractive index) is to be measured.

$$
\theta=\frac{2 \pi}{\lambda} \int_{0}^{L} \Delta n(z) d z
$$

In case of heterogeneous source such measurements are to be done for different directions of plasma probing. In tasks of diagnostics of plasma the most convenient methods are holographic interference methods since there is no need to take care of interference quality of plasma bordering surfaces and of tuning of interferometer. Students study the principles of holography, main methods of holographic interference: method of two exposures and method of "alive bands", allowing to dynamically observe changes of refractive index of nonstationary objects. As exercises they are offered to calculate the minimal densities of atoms and electrons, which are possible to measure by the holography method under given discharge geometry and noise level of a system, measuring intensity of interference pattern.

In section "diagnostics based on light scattering" there have been considered on the basis of classical electrodynamics the possibility of use of line shape of Thomson scattering on free electrons for study of electron velocities distribution functions, as well as criteria for applicability of this method are specified. If these criteria are not complied (at large electronic densities and small scattering angles), information on plasma parameters is contained in scattering spectrum in other form: combinational scattering on plasma fluctuations occurs. Frequency of fluctuations is connected with density and temperature of electrons. In this section principle of Coherent Antistokes Raman Spectroscopy and its use for determination of populations of levels of atoms and molecules are considered also. Developing within the framework of REC direction of dust plasma studies has required introduction in the program of methods of dusty particles diagnostics by light scattering. In particular, in student's educational and research works different software realizations of theory of Mie were executed [13].

The common way of registering spatial distribution of heterogeneous plasma parameters basing on the integral characteristics of source (radiance of surface, optical thickness, phase difference) is to implement the tomography. So the study of the main terms of tomography (cross section and projection), mathematical basis of computer tomography (Radon's transform and Abel's transform as its private case), use of Fourier transformations in tomography and description of main methods of reconstruction of cross sections basing on projections are included in the program of this course. Some of these techniques are reviewed in training program on tomography, which is presented in another report entitled "The computer training program on tomography". These methods are also introduced in few student works. In particular in one of the diploma works tomographic interferometer was constructed [14], what in fact is an analog processor design. It realizes the method of inverse projection for 
reconstruction of lines of equal refractive index, i.e. of equal density of atoms, basing on phase shifts of waves illuminating heterogeneous object from different directions (fig. 4).

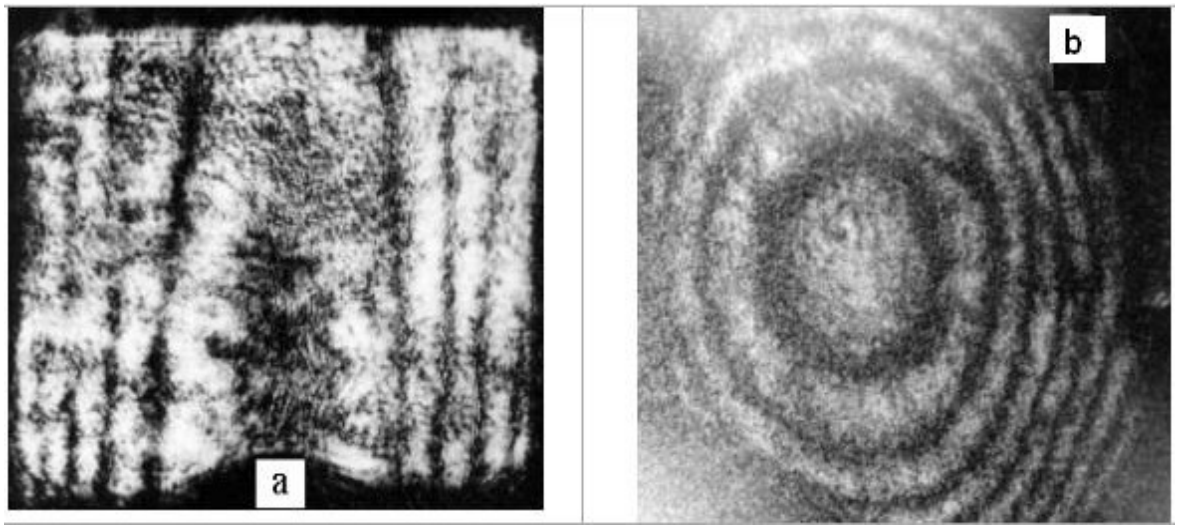

Fig. 4. a). interferogram of flame of a candle (one projection); b). reconstructed by analog processor cross section x,y; lines of equal refractive index are observed.

Application of tomography to study of dusty plasma is described in the work, executed in collaboration with student and submitted to Northern Optics 2003 conferences [15].

A rational algorithm of data processing for wide-spread case of axial symmetric heterogeneous source is also in details considered in course [16,17].

For investigation of one section of cylindrically symmetric plasma the two-dimensional array of samples $\mathrm{F}\left(\lambda_{\mathrm{i}}, \mathrm{x}_{\mathrm{j}}\right)$ corresponding to spectral surface radiance $\mathrm{b}\left(\lambda_{\mathrm{i}}, \mathrm{x}_{\mathrm{j}}\right)$ have to be measured along a series of chords, perpendicular to plasma axis $\left(\mathrm{x}_{\mathrm{j}}\right.$ - the displacement of chord from plasma centerline, $\lambda_{i}$ - wavelength of spectral point inside the profile). From the array $b\left(\lambda_{\mathrm{i}}, \mathrm{x}_{\mathrm{j}}\right)$ the array of spectral emissivities $\varepsilon\left(\lambda_{\mathrm{i}}, \mathrm{r}_{\mathrm{j}}\right)$ has to be reconstructed and spectral line profiles $\varepsilon_{j}\left(\lambda_{i}\right)$ for distance $r_{j}$ from the section center have to be found. These profiles may be used for the determination of plasma parameters in this spatial point. The reconstruction of emissivity $\varepsilon(\mathrm{r})$ from measured intensity $\mathrm{b}(\mathrm{x})$ is known as Abel's inversion

$$
\varepsilon_{\lambda}(r)=-\frac{1}{\pi} \int_{r}^{R_{0}} \frac{d b_{\lambda}(x)}{d x} \frac{d x}{\sqrt{\left(x^{2}-r^{2}\right)}} .
$$

Here $\mathrm{R}_{0}$ is the discharge tube radius.

It is so called improperly posed problem and the solution is impossible without using of a priory information about function $\varepsilon(\mathrm{r})$. But measured line profiles $\mathrm{F}\left(\lambda_{\mathrm{i}}, \mathrm{x}_{\mathrm{j}}\right)$ may be distorted by the spectrometer spread function.

$$
F_{\text {out }}(\lambda)=\int F_{\text {in }}\left(\lambda^{\prime}\right) \cdot g\left(\lambda-\lambda^{\prime}\right) d \lambda^{\prime} .
$$

$\mathrm{g}(\lambda)$ is the spectrometer spread function, $F_{\text {out }}$ is the observed line profile, $F_{\text {in }}$ is the true line shape and it is proportional to $b(\lambda)$. The elimination of instrument distortion is possible, for example, by use of the convolution theorem [like equation (3)]. If $g(\lambda)$ is known we simply have to divide Fourier transform of $F_{\text {out }}$ by Fourier transform of $g$ and then reconstruct $F_{\text {in }}$ by the inverse Fourier transform of the result of division. But it is also an improperly stated problem, its solving is very sensitive to experimental data noises. The algorithm describe in this course significantly increases the results stability in the presence of experimental data noises, when it is necessary to solve both improperly stated problems concurrently. The algorithm is based on collective processing of large data arrays of sample data $\mathrm{F}\left(\lambda_{\mathrm{i}}, \mathrm{x}_{\mathrm{k}}\right)$.

For the first step of algorithm one has to calculate the covariance matrix A of the array of profiles $F\left(\lambda_{i}, x_{k}\right)=F_{i k}$, corresponding to one or, perhaps, several spectral lines and time points.

$$
A_{k, j}=\frac{1}{n} \sum_{i=1}^{n}\left(F_{i, k}-\bar{F}_{k}\right)\left(F_{i, j}-\bar{F}_{j}\right) \text {, }
$$

where $\mathrm{n}$ is a number of spectral points, $\overline{F_{k}}=\frac{1}{n} \sum_{i=1}^{n} F_{i, k}$ is an average spatial vector.

Then the array $\mathrm{F}_{\mathrm{ik}}$ may be described by a model: 


$$
F_{i, k}=\bar{F}_{k}+\sum_{p=1}^{q} M_{i, p} U_{p, k}
$$

where $U_{p}$ are eigenvectors of the covariance matrix $A$ and $M_{i, p}$ are the projections of $i$-th spectral point to eigenvector $U_{p}$.

$$
M_{i, p}=\sum_{k=1}^{m}\left(F_{i, k}-\bar{F}_{k}\right) U_{p, k}
$$

As a consequence of mutual dependence of elements $\mathrm{F}_{\mathrm{ik}}$ the number $\mathrm{q}$ of significant eigenvectors of $\mathrm{A}$ is less than $\mathrm{m}$. The eigenvector is significant if corresponding eigenvalue is no less than estimator $\mathrm{S}^{2}$ of variance of experimental error. Before further processing one has to prove that model (5) with $\mathrm{m}(\mathrm{q}+1)+\mathrm{qn}$ coefficients is adequate. For this purpose the Fisher criterion has to be used [18].

The operations of radial transform (let us denote the corresponding operator $\boldsymbol{R}$ ) and elimination of instrument distortion (let us denote the corresponding operator $\boldsymbol{C}$ ) are linear and applied to different variables. So instead of the instrument distortion elimination from each profile $\mathrm{F}_{\mathrm{k}}(\lambda)$, and then Abel's inversion for each wave length $\lambda_{\mathrm{i}}$ for the next steps of algorithm one can eliminate the instrument distortions from projection $\mathrm{M}_{\mathrm{i}, \mathrm{p}}$, then fulfill the radial transform for $\overline{\mathrm{F}}$ and $\mathbf{U}_{\mathrm{p}}$ and restore the required profiles $\varepsilon\left(\lambda_{\mathrm{i}}, \mathrm{r}_{\mathrm{j}}\right)$ in various plasma points $\mathrm{r}$ as:

$$
\varepsilon(\lambda, \mathrm{r})=\boldsymbol{R}\{\overline{\mathrm{F}}\}+\sum_{\mathrm{p}=1}^{\mathrm{q}} \mathrm{L}_{\mathrm{p}}(\lambda) \boldsymbol{R}\left\{\mathrm{U}_{\mathrm{p}}\right\},
$$

where $\mathrm{L}_{\mathrm{ip}}=\boldsymbol{C}\left\{\mathrm{M}_{\mathrm{i}, \mathrm{p}}\right\}$ are the results of instrument distortion elimination from parts of projections $\mathrm{M}_{\mathrm{i}, \mathrm{p}}$, corresponding to each spectral line.

The spectroscopic method of determination of electric field strength in plasma is considered in the final section of course. This is very important in particular in investigations of dusty plasma, when using the standard probe method is impossible. At the moment two principles of using laser spectroscopy are offered to measure the local fields in plasma.

The First method is based on the measurement of Stark splitting higher atomic levels. In one variant using tunable laser the population of certain Stark sublevel of one of higher excited states is increased and the fact of increase is registered by means of optogalvanic effect - the discharge current growth due to increase of number of ionizations from this level [19]. In another variant [20] transition to Stark sublevel causes the reduction of population of lower level, the decrease corresponds to decrease of intensity on fluorescence lines from this devastated level.

The Second method is based on appearance of prohibited lines in spectra of atoms or molecules in the presence of external electric field since wave functions of states in external field are described by the superposition of different states and selection rules legal for fields of central symmetry are broken. Superposition coefficients are calculated by the solving appropriate Shrödinger's equation and the relationship of intensity of prohibited line with field strength is revealed [21]. This result has great cognitive importance, illustrating practical profit of quantum mechanics technique.

\section{Laboratory programs on optics and spectroscopy}

At study of course of general physics students execute the standard set of laboratory programs, illustrating the laws of geometric optics, phenomena of light interference, polarization, diffraction as well as optical pyrometry. In course of laser physics works on measurement of spectral composition, power and angular divergence of laser radiation are fulfilled. In course "Physical bases of reception of information" all students get acquainted with process of registering and recovering holograms and execute simple experiments on optical spatial filtration, in particular, installing and moving the "knife" in Fourier plane of a system, drawing image of an object, and visualize the defects in transparent objects, evaluate the sizes of heterogeneous areas in objects. These programs prepare the specialists to use the holographic and shadow methods of plasma diagnostics.

The majority of works in the scope of this course as well as of courses on atomic and quantum physics spectroscopy and plasma diagnostics are carried out with use of new information technologies and a possibility of remote access to the equipment. Students come to know a subject area (e.g. determination of fundamental atomic constants, Zeeman effect, plasma diagnostics, temperature determination, eliminating the apparatus distortions etc.) in parallel with acquiring habits in usage of modern information systems and effective methods of accumulation, processing and transformation of data. They are trained in usage of local and remote information resources and study the operation principles and possibilities of various measurement sensors [22].

Hardware and software for these laboratory programs are based on LabView and compatible instrument interfaces. It is built of separated modules and can be easily adapted for any set of the spectral equipment and any modern operational environment. Each module is made as a "virtual instrument", on the panel of which there are 
windows for input of the necessary information, control buttons (start of the module, save file) and graphic field for result representation. Examples of such panels are given in figures 5, 6.

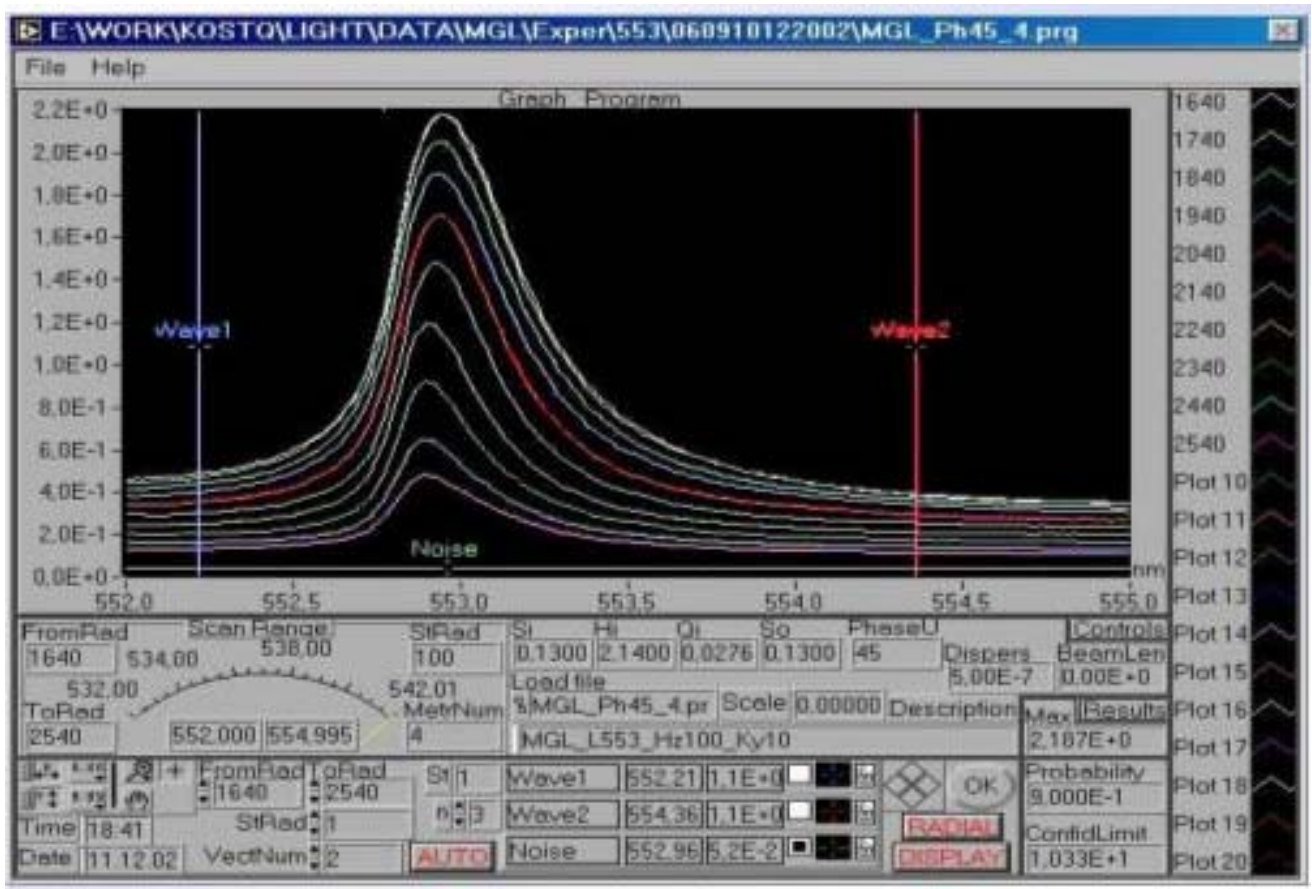

Fig. 5. Laboratory program "Emission spectroscopy of heterogeneous plasma". Array of spectral line profiles $b\left(\lambda_{\mathrm{i}}, \mathrm{x}_{\mathrm{j}}\right)$ for different position of spatial scanning system.

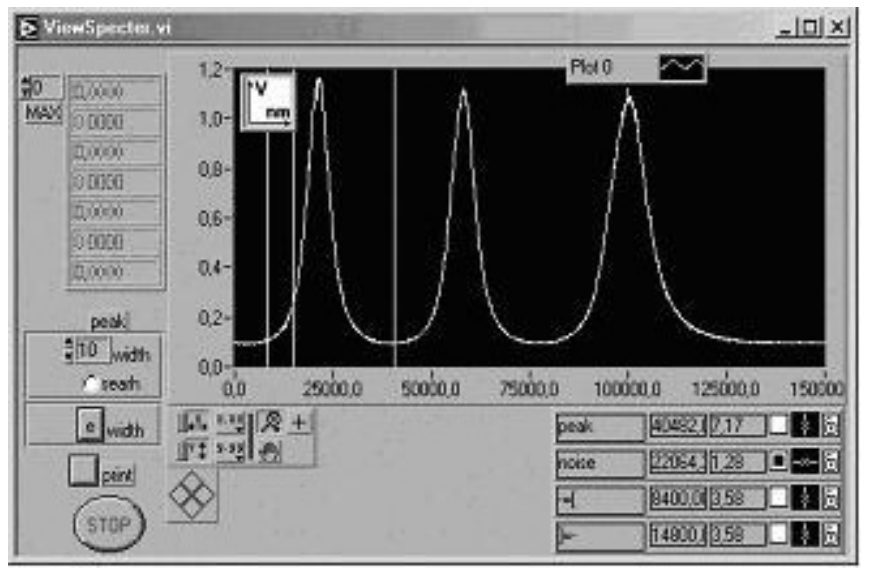

Fig. 6. Laboratory program "Determination of atom temperature by spectral line broadening". Spectral line shape obtained by scanning Fabry-Perot interferometer before elimination of instrument distortion.

By processing the array presented in Fig. 7 using the above described algorithm students have to receive spectral line profiles $\varepsilon_{j}\left(\lambda_{i}\right.$, ) for different distances $r_{j}$ from the discharge center and to estimate electron or atom density at this point by spectral line width or shift [1].

For processing data presented in Fig.8 students have to calculate the interferometer spread function:

$$
P\left(\beta \left(=\frac{(1-R)^{2}}{(1-R)^{2}+4 R \sin ^{2} \pi \beta}\right.\right.
$$


( $\beta$ is the fraction of the distance between the nearby interference peaks, $\mathrm{R}$ - mirror reflectance) and to eliminate the instrument distortion by free accessed program [23].

It should be mentioned that almost all our spectroscopic installations allow the work in distributed research nets and provide remote access to information and physical equipment through Intranet/Internet channels.

The knowledge obtained in the discussed courses is also supported in special lecture and practical course "Automation of scientific research".

Besides studying basic subjects students can choose an optional course, for example, "Nonlinear optics" or "Optical processors". The latter is devoted to the principles of Fourier optics, their application to certain problems of processing and scene analysis, including the possibility of using spatial filtrations for spectrum decryption and exclusion of apparatus distortions [24], as well as to the principles of integral optics, acoustic optics, optical bistable and logical elements, realization of optical relationships in neurocomputers and matrix elements (optical programmable logic arrays). The principles and perspectives of quantum computers are considered. It spreads the horizon of students, makes the perception of new ideas in the theme field of REC "Plasma" easier (for example, recent propose to use optoacoustic filter as an element of image spectrometer) [25].

All students use web-based textbooks, learning software (e.g. "Fourier spectrometer", "Modeling and exclusion of apparatus distortion") and other web-resources published on site of department (http://dfe3300.karelia.ru/) and on REC's site (http://plasma.karelia.ru/). One of the subdivisions of the latter site contains lectures on plasma diagnostics read at summer schools on low temperature plasma physics and at scientific seminars of REC "Plasma".

\section{References}

[1] H. R. Griem, Plasma Spectroscopy (New York: MCGraw-Hill, 1964).

[2] Plasma diagnostics. Edited by W Lochte- Holtgreven (North-Holland Publishing company, Amsterdam, 1968).

[3] I.I. Sobelman, Introduction to the theory of atomic spectra (Moscow, Fizmatgiz, 1963).

[4] Photon correlation and light beating spectroscopy. Edited by H.Z. Cummins and E. R. Pike (Plenum press, New York and London, 1974).

[5] O.S. Vaulina, S. V. Vladimirov, "Diffusion and dinamics of macro-particles in a complex plasma" in Physics of Plasmas, (9, 2002), pp. 835-840.

[6] A. Khakhaev, L. Luizova, S Podryadchikov, A.Scherbina and A. Shtykov, "Experimental set up for investigation of ordered dusty structures in the glow discharge" in International conference on physics of low temperature plasma PLTP-03 (Kyiv, Ukraine, May 11-15, 2003), in press.

[7] W. Demtroder, Laser spectroscopy, (Springer-Verlag, Berlin, New York, 1982).

[8] G.L. Klimchitskaya, I.N. Polushkin, E.A. Sviridenkov, Intraresonator laser diagnostics of plasma. (Moscow: Energoizdat, 1994).

[9] W. R. Bennet, "Effect of gaps on laser lines on He-Ne mixture" in Phys. Rev. (1962, V.126, № 2), p. 580.

[10] L.S. Vasilenko, L.N. Guskov et al, Laser systems (Novosibirsk, 1980), pp. 128-139.

[11] A.M. Badalyan, M.E. Movsesyan, V.O. Chaltykyan, in Optics and spectroscopy (1994, V. 76, issue 6), pp. 900-903.

[12] NIST Atomic Spectra Database, http://physics.nist.gov/cgi-bin/AtData/main asd

[13] K. Boren, D. Hofman, Absorption and scattering of light by small particles, (Moscow: Mir, 1986).

[14] G.I. Vishnyakov, G.G. Levin, "Optical tomography of plasma objects" in Optics and spectroscopy (1982, V. 53, issue 4), pp. 731-736.

[15] A.V. Bulba, L.A. Luizova, A.D. Khakhaev, "Dusty plasma tomography" in Northen Optics 2003 (16-18 June, Espoo, Finland), p. 49.

[16] L.A. Luizova, "Abel's inversion applied to array of spectral line profiles" in JQRST (66, 277 2000).

[17] L. Luizova, A. Khakhaev, K. Ekimov and A. Soloviev, "Rational tools for data obtaining and processing in local plasma spectroscopy" in Abstracts of 16 Symposium on Plasma Chemistry (Taormina, Italy, June 22-27, 2003), p. 107.

[18] J. E. Jackson, A User's Guide To Principal Component (New York: John Willey \&Sons, 1991).

[19] V.P. Gavrilenko, "Spectrscopic Method for Measurement of Electric Field in Plasma" in Spectra Line Shapes (V 10, 14th ICSL, AIP Conf. Proc. 467, New York, 1998), pp. 14-26.

[20] E.R. Chercasova, V. P. Gavrilenko, A.I. Zhuzhunashvili, "Spectroscopic Measurement of Electric Field in a Hollow Cathode Discharge and a Beam-Plasma Discharge" in Abstracts of 16 Symposium on Plasma Chemistry, (Taormina, Italy, June 22-27, 2003), p. 61.

[21] U. Czarnenzki, "Plasma diagnostics by Electric Field Measurement" in 16 Abstracts of Symposium on Plasma Chemistry, (Taormina, Italy, June 22-27, 20030, p.52.

[22] K.A. Ekimov, R.V. Prichodchenko, A.D. Khakhaev, "Laboratory programs on physics with usage of modern information technologies" in International Conference on Engineering and Computer Education ICECE2003 (Brazil, San-Paolo, 2003), in press.

[23] L.A. Luizova, A.V. Soloviev, "Computer Training Program for Elimination of Instrument Distortion" in $7^{\text {th }}$ International Conference on Education and Training in Optics and Photonics (2001, Proc. SPIE, 4588, 440, 2002).

[24] L.A. Luizova, A.D. Khakhaev, V.P. Chugin, "Use of optical spatial filtration in spectroscopy" in Optics and spectroscopy (1975, V. 39, issue 2), pp. 765-767.

[25] S. Marke, Th. Oberreuther, N. Wallendorf, Ch. Wolf, "Imaging emission spectroscopy jf atmospheric pressure Carbon dioxide microwave discharge using an Acousto Optical Tunable Filter" in Abstracts of 16 Symposium on Plasma Chemistry (Taormina, Italy, June 22-27, 20030, p. 108. 